\author{
Marquette University \\ e-Publications@Marquette
}

4-2009

\title{
Variation of benzyl anions in MgAl-layered double hydroxides: Fire and thermal properties in PMMA
}

Calistor Nyambo

Marquette University

Dan Chen

Hunan Normal University

Shengpei Shu

Hunan Normal University

Charles A. Wilkie

Marquette University, charles.wilkie@marquette.edu

Follow this and additional works at: https://epublications.marquette.edu/chem_fac

Part of the Chemistry Commons

\section{Recommended Citation}

Nyambo, Calistor; Chen, Dan; Shu, Shengpei; and Wilkie, Charles A., "Variation of benzyl anions in MgAllayered double hydroxides: Fire and thermal properties in PMMA" (2009). Chemistry Faculty Research and Publications. 89.

https://epublications.marquette.edu/chem_fac/89 
Marquette University

e-Publications@Marquette

\section{Chemistry Faculty Research and Publications/College of Arts and Sciences}

This paper is NOT THE PUBLISHED VERSION; but the author's final, peer-reviewed manuscript. The published version may be accessed by following the link in th citation below.

Polymer Degradation and Stability, Vol. 94, No. 4 (April 2009): 496-505. DOI. This article is (C Elsevier and permission has been granted for this version to appear in e-Publications@Marquette. Elsevier does not grant permission for this article to be further copied/distributed or hosted elsewhere without the express permission from Elsevier.

\section{Variation of Benzyl Anions in MgAl-Layered Double Hydroxides: Fire and Thermal Properties in PMMA}

\section{Calistor Nyambo}

Department of Chemistry and Fire Retardant Research Facility, Marquette University, Milwaukee, WI Dan Chen

College of Chemistry and Chemical Engineering, Hunan Normal University, Changsha, Hunan 410081, People's Republic of China

Shengpei Su

College of Chemistry and Chemical Engineering, Hunan Normal University, Changsha, Hunan 410081, People's Republic of China

Charles A. Wilkie

Department of Chemistry and Fire Retardant Research Facility, Marquette University, Milwaukee, WI

\section{Abstract}

Magnesium aluminum layered double hydroxides (MgAl-LDHs) intercalated with a range of benzyl anions were prepared using the coprecipitation method. The benzyl anions differ in functionality (i.e. carboxylate, sulfonate, 
and phosphonate) and presence or absence of an amino substituent. Various methods for preparing LDHs (i.e. ion exchange, coprecipitation and rehydration of the calcined LDH methods) have been compared with the MgAl-benzene phosphonate and their effect on fire and thermal properties was studied. After characterization, the MgAl-LDHs were melt-blended with poly(methyl methacrylate) (PMMA) at loadings of 3 and $10 \%$ by weight to prepare composites. Characterization of the LDHs and the PMMA composites was performed using FTIR, XRD, TGA, transmission electron microscopy (TEM) and cone calorimetry. FTIR and XRD analyses confirmed the presence of the charge balancing benzyl anions in the galleries of the MgAl-LDHs. Improvements in fire and thermal properties of the PMMA composites were observed. The cone calorimeter revealed that the addition of $10 \% \mathrm{MgAl}-\mathrm{LDH}$ s reduces the peak heat release rate by more than $30 \%$.

\section{Keywords}

Poly(methyl methacrylate), Layered double hydroxide, Thermogravimetry, Cone calorimeter, Fire retardancy

\section{Introduction}

Layered double hydroxides (LDHs) are interesting anionic clays that may play an important part in the development of fire retardant systems for polymers, because the addition of LDH has been shown to enhance thermal stability and improve fire properties [1], [2], [3]. In addition, it has been observed that LDHs show some synergistic effects with commercial flame retardants, like ammonium polyphosphate (APP), giving rise to improvements in fire retardancy performance ${ }^{[4]}$.

LDHs consist of layers of divalent $\left(\mathrm{M}^{2+}\right)$ and trivalent $\left(\mathrm{M}^{3+}\right)$ cations co-ordinated octahedrally by hydroxyl groups. Their structure is similar to that of brucite, $\mathrm{Mg}(\mathrm{OH})_{2}$, in which isomorphous replacement of the divalent cation with trivalent cation creates a positive charge on the metal layers which is counter balanced by the presence of anions in the galleries $[5]$. Thus LDHs have a reverse polarity from cationic clays with regard to layer and interlayer anion charge. Their chemical structure is represented by the formula; $\left.\left[\mathrm{M}^{2+}{ }_{1-x} \mathrm{M}^{3+}{ }_{x}(\mathrm{OH})_{2}\right]^{x+}\left(\mathrm{A}_{x / n}\right)^{n-} \cdot \mathrm{mH}_{2} \mathrm{O}\right]$, where $\mathrm{M}^{2+}=\left(\mathrm{Mg}^{2+}, \mathrm{Zn}^{2+}\right.$, or $\left.\mathrm{Ni}^{2+}\right), \mathrm{M}^{3+}=\left(\mathrm{Al}^{3+}, \mathrm{Fe}^{3+}, \mathrm{Mn}^{3+}\right.$ etc. $)$ and $\mathrm{A}^{n-}$ is the charge balancing interlayer anion. Like cationic clays, LDH can be organically modified. This is easily achieved by intercalating charge balancing organic anions with different functionalities, such as carboxylate, sulfonate, phosphonate and others. It is important to understand the effect of varying functionalities of organic anions in LDHs to be able to optimize improvements in thermal and fire properties.

Since LDHs are synthetic materials, it is possible to study the effect on fire and thermal properties of various methods of preparation. The commonly used methods of preparing LDHs are: (i) ion exchange of a precursor LDH intercalated with a nitrate or chloride, (ii) direct synthesis, i.e., the coprecipitation method, in which metal nitrates or chlorides are introduced to a solution of the organic anion at a controlled $\mathrm{pH}$, (iii) thermal or melt reaction of $\mathrm{MgAl}$-carbonate LDHs and organic guest anion by heating at low ramp rate up to temperatures $10-20^{\circ} \mathrm{C}$ above the melting point of organic acid and (iv) rehydration of the calcined LDH in the presence of water and organic anion [6], [7], [8], [9], [10], [11]. A swelling agent such as glycerol may be used during

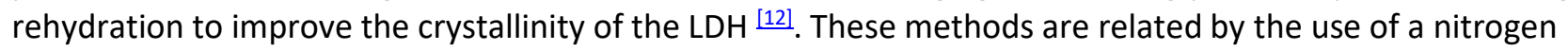
atmosphere, decarbonated water and deionized water, to minimize contamination by carbon dioxide which would lead to the formation of a carbonate in the LDH. Nevertheless, contamination by carbonate is difficult to avoid.

The aim of this work is to study the effect of the mode of preparation for a range of benzyl anions with different functionalities (carboxylate, sulfonate and phosphonate) and amino substituent in MgAl-LDHs on the fire and thermal properties in PMMA composites.

\section{Experimental}

\subsection{Materials}

Poly(methyl methacrylate), PMMA, $M_{w} 120,000$, benzoic acid $\left(\mathrm{C}_{7} \mathrm{H}_{6} \mathrm{O}_{2}\right)$, 4-amino benzoic acid $\left(\mathrm{C}_{7} \mathrm{H}_{7} \mathrm{NO}_{2}\right)$, sodium hydroxide flakes, sodium nitrate, glycerol, magnesium nitrate hexahydrate, $\mathrm{Mg}\left(\mathrm{NO}_{3}\right)_{2} \cdot 6 \mathrm{H}_{2} \mathrm{O}$, aluminum 
nitrate nonahydrate, $\mathrm{Al}\left(\mathrm{NO}_{3}\right)_{3} \cdot 9 \mathrm{H}_{2} \mathrm{O}$ were obtained from Aldrich Chemical Company while benzene sulfonic acid, $\left(\mathrm{C}_{6} \mathrm{H}_{5} \mathrm{SO}_{3} \mathrm{H} \cdot \mathrm{H}_{2} \mathrm{O}\right.$,) and benzene phosphonic acid $\left(\mathrm{C}_{6} \mathrm{H}_{5} \mathrm{PO}(\mathrm{OH})_{2}\right)$ were acquired from TCl. The hydrotalcite, (pural $\mathrm{MG63HT)}$, referred to herein as MgAl-carbonate LDH, was kindly provided by Sasol Germany GmbH.

\subsection{Instrumentation}

Powder X-ray diffraction measurements (PXRD) and XRD for the composites were performed with a Rigaku, Miniflex II Desktop, X-ray diffractometer with Cu (K alpha) radiation $\lambda=1.54078 \AA$, from a sealed X-ray tube. The powder sample was prepared and mounted on a glass holder, while the composites were prepared by compression molding and mounted on aluminum sample holder. The data were collected at various $2 \vartheta$ values from $0^{\circ}$ to $70^{\circ}$ at a scan speed of $5^{\circ}$ per minute with a sampling width of 0.02 . Basal spacing of LDHs and ethylene vinyl acetate nanocomposites were obtained from the $00 /$ reflections.

Bright field transmission electron microscopy (TEM) images of the nanocomposites were obtained at $60 \mathrm{kV}$ with a JEOL 1230 electron microscope. The samples were ultramicrotomed with a diamond knife on AO-E microtome at room temperature to give $\sim 90 \mathrm{~nm}$ thick section. The sections were then transferred from the knife-edge to Cu grids.

Fourier transform infrared spectra were obtained using the $\mathrm{KBr}$ method on a Nicolet Magna-IR 560 spectrometer operated at $1 \mathrm{~cm}^{-1}$ resolution in the $400-4000 \mathrm{~cm}^{-1}$ region. Thermogravimetric analysis (TGA) and differential thermal analysis (DTA) were performed on a SDT 2960 simultaneous DTA-TGA instrument from 50 to $800{ }^{\circ} \mathrm{C}$ at a heating rate of $20^{\circ} \mathrm{C} / \mathrm{min}$ in $\mathrm{N}_{2}$, flowing at $85 \pm 5 \mathrm{~mL} / \mathrm{min}$, with sample sizes of $15.0 \pm 1.0 \mathrm{mg}$ contained in aluminum sample cups. All samples were run in triplicate and the average values are reported; temperatures are considered accurate to $\pm 3{ }^{\circ} \mathrm{C}$ and the error on the fraction of non-volatile materials is $\pm 2 \%$. Calcination was performed in a Thermolyne 1300 Furnace at $450{ }^{\circ} \mathrm{C}$ for $18 \mathrm{~h}$ for the hydrotalcite and at $1000^{\circ} \mathrm{C}$ for $24 \mathrm{~h}$ for the char obtained after cone calorimetry.

Approximately $30 \mathrm{~g}$ of poly (methyl methacrylate) composite samples were compression molded into $10 \mathrm{~cm} \times 10 \mathrm{~cm}$ square plaques of uniform thickness $(\sim 3 \mathrm{~mm})$ before cone calorimetry was performed on an Atlas Cone 2 instrument according to ASTM E 1354 at an incident flux of $50 \mathrm{~kW} / \mathrm{m}^{2}$ with a cone shaped heater; the spark was continuous until the sample ignited. All samples were run in triplicate and the average value, with standard deviation, is reported; results from cone calorimeter are generally considered to be reproducible to $\pm 10 \%$.

\subsection{Preparation of magnesium aluminum layered double hydroxides by coprecipitation}

The magnesium aluminum layered double hydroxides (MgAl-LDHs) intercalated with benzyl anions were prepared following a literature procedure $\stackrel{[13]}{ }$. A solution of $\mathrm{Mg}\left(\mathrm{NO}_{3}\right)_{2} \cdot 6 \mathrm{H}_{2} \mathrm{O}(0.02 \mathrm{~mol})$ and $\mathrm{Al}\left(\mathrm{NO}_{3}\right)_{3} \cdot 6 \mathrm{H}_{2} \mathrm{O}$ $(0.01 \mathrm{~mol})$ in deionized and decarbonated water $(50 \mathrm{ml})$ was added dropwise to a solution of the organic acid $(0.02 \mathrm{~mol})$ (i.e. benzoic acid, (BA), benzene sulfonic (BS), 4-amino benzoic (ABA), or benzene phosphonic (BP)), with the molar equivalent of $\mathrm{NaOH}$ in deionized and decarbonated water $(100 \mathrm{ml})$ with vigorous mixing under an inert nitrogen atmosphere. The $\mathrm{pH}$ was maintained at 10.0 by adding $1 \mathrm{M} \mathrm{NaOH}$ solution. The resultant slurry was aged at $60^{\circ} \mathrm{C}$ for $24 \mathrm{~h}$, cooled to room temperature, and repeatedly washed with deionized and decarbonated water before drying under an inert atmosphere at a temperature of $50^{\circ} \mathrm{C}$. The chemical structures of the benzyl anions are shown in Fig. 1.

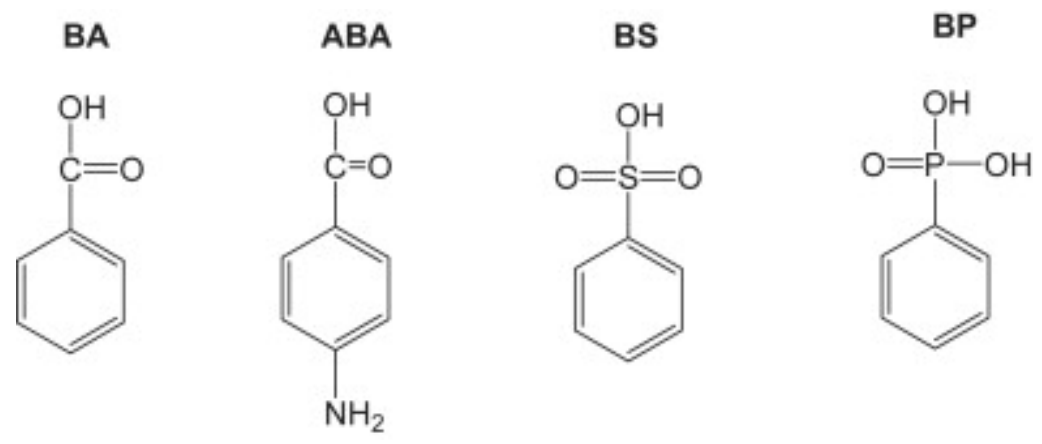

Fig. 1. Chemical structures of the charge balancing anions used to prepare the MgAl-LDHs. 


\subsection{Preparation of magnesium-aluminum nitrate layered double hydroxide}

The magnesium-aluminum nitrate layered double hydroxide (MgAl-nitrate LDH) was synthesized by the

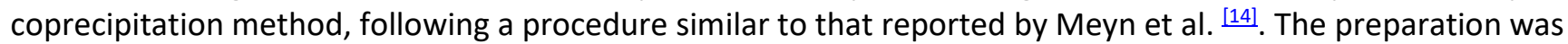
performed in a nitrogen atmosphere to exclude $\mathrm{CO}_{2}$ which would lead to the incorporation of carbonate in the LDHs. A solution of $32.0 \mathrm{~g}$ of $\mathrm{Mg}\left(\mathrm{NO}_{3}\right)_{2} \cdot 6 \mathrm{H}_{2} \mathrm{O}(0.125 \mathrm{~mol})$ and $23.4 \mathrm{~g}$ of $\mathrm{Al}\left(\mathrm{NO}_{3}\right)_{3} \cdot 9 \mathrm{H}_{2} \mathrm{O}(0.0625 \mathrm{~mol})$ in $125 \mathrm{ml}$ of degassed and deionized water was added dropwise over $1 \mathrm{~h}$ to a solution of $12.5 \mathrm{~g}$ of $\mathrm{NaOH}(0.313 \mathrm{~mol})$ and $18.2 \mathrm{~g}$ of $\mathrm{NaNO}_{3}(0.214 \mathrm{~mol})$ in $145 \mathrm{ml}$ of degassed/deionized water. The $\mathrm{pH}$ of the solution was maintained at 10.0 by adding $1 \mathrm{M} \mathrm{NaOH}$ solution as needed. The resulting white precipitate was aged for $24 \mathrm{~h}$ at $65^{\circ} \mathrm{C}$, then filtered until all of the supernatant liquid was removed. The sample was washed several times with large amounts of deionized and degassed water, and was dried at $50^{\circ} \mathrm{C}$ in a vacuum oven.

\subsection{Preparation of magnesium-aluminum benzene phosphonate layered double hydroxide by anion exchange}

The magnesium-aluminum phosphonate layered double hydroxide (referred to herein as MgAl-BP LDH exchange) was prepared from the $\mathrm{MgAl}-\mathrm{NO}_{3} \mathrm{LDH}$ using an ion exchange reaction. In a typical preparation, $5 \mathrm{~g}$ of the $\mathrm{MgAl}-\mathrm{NO}_{3} \mathrm{LDH}$ was dispersed in $100 \mathrm{ml}$ of a $0.008 \mathrm{M}$ anion solution prepared by dissolving the benzene phosphonic acid in warm water containing $0.016 \mathrm{M}$ of $\mathrm{NaOH}$ under a steady flow of nitrogen to exclude carbon dioxide. The solution was vigorously stirred for $24 \mathrm{~h}$ at $60^{\circ} \mathrm{C}$ and the solid was separated, washed with degassed/deionized water and dried at $50{ }^{\circ} \mathrm{C}$. The ion exchange was repeated several times and completion of exchange was ascertained by the absence of the $1384 \mathrm{~cm}^{-1} \mathrm{NO}_{3}{ }^{-}$band in the IR spectra of the MgAl-LDHs.

\subsection{Preparation of magnesium-aluminum phosphonate layered double hydroxide by rehydration}

The magnesium-aluminum benzene phosphonate LDH (referred hereafter as MgAl-BP LDH rehydration) was prepared by rehydration of the calcined hydrotalcite following a procedure reported by Dimotakis and Pinnavaia ${ }^{[12]}$. In a typical experiment, the MgAl-carbonate LDH was calcined in air at $450 \pm 10^{\circ} \mathrm{C}$ for $18 \mathrm{~h}$. $1 \mathrm{~g}$ of the calcined material was then added to a $100 \mathrm{ml}$ of $0.1 \mathrm{M}$ benzene phosphonate solution prepared by dissolving the benzene phosphonic acid in warm water/glycerol solution (1:2 volume ratio), degassed and deionized water containing $0.2 \mathrm{M}$ of $\mathrm{NaOH}$ under a steady flow of nitrogen to exclude carbon dioxide at $50{ }^{\circ} \mathrm{C}$ and aged for $24 \mathrm{~h}$. The resulting slurry was then filtered and washed several times with hot deionized and degassed water, and then dried at $50^{\circ} \mathrm{C}$ in a vacuum oven.

\subsection{Preparation of the poly(methyl methacrylate) composites}

Poly(methyl methacrylate)-LDH composites were prepared via melt blending using established methods $\frac{[15]}{}$. Poly(methyl methacrylate) composites were prepared by melt blending in a Brabender mixer (temperature $=190^{\circ} \mathrm{C}$, screw speed $=60 \mathrm{rpm}$, and time $=8 \mathrm{~min}$ ). The LDH loadings were 3 and $10 \%$ by weight. $A$ reference sample of unmodified poly(methyl methacrylate) was obtained by following the same procedure without any additive.

\section{Results and discussion}

\subsection{Characterization of MgAl-LDH and its PMMA composites}

\subsubsection{Infrared and X-ray diffraction analysis of the MgAl-LDHs}

Fourier transform infrared spectroscopy (FTIR) was used to ascertain the presence of the benzyl anions in the LDHs; the FTIR spectra are presented in Fig. 2(i) and an expansion showing the fingerprint region is in Fig. 2(ii). All the MgAl-LDHs showed a broad band around $3400 \mathrm{~cm}^{-1}$ characteristic of the $\mathrm{OH}$ stretching vibration arising from the metal hydroxyl groups and hydrogen bonded interlayer water molecules. Bands around $1448 \mathrm{~cm}^{-1}, 1487 \mathrm{~cm}^{-1}, 1468 \mathrm{~cm}^{-1}$ and $1602 \mathrm{~cm}^{-1}$ are assigned to the $\mathrm{C}=\mathrm{C}$ vibrations from the aromatic rings of the charge balancing anions. With the MgAl-BA and MgAl-ABA, peaks at $1557 \mathrm{~cm}^{-1}$ and $1412 \mathrm{~cm}^{-1}$ are assigned to the vibration of the carboxylate, while the presence of the $\mathrm{N}-\mathrm{H}$ bend at $1612 \mathrm{~cm}^{-1}$ in the $\mathrm{MgAl}-\mathrm{ABA}$ 
is the only visible peak that distinguishes it from MgAl0-BA LDH. The MgAl-BP, showed vibrations between $1200 \mathrm{~cm}^{-1}$ and $1000 \mathrm{~cm}^{-1}$ from $-\mathrm{PO}_{3}-$. As might be expected, there was no difference in the FTIR spectra for the MgAl-BP prepared using different methods. The strong peaks appearing at $1350 \mathrm{~cm}^{-1}$ and $1180 \mathrm{~cm}^{-1}$ in the spectrum for MgAl-BS are associated with the asymmetric and symmetric vibrations of $\mathrm{S}-\mathrm{O}$, respectively.
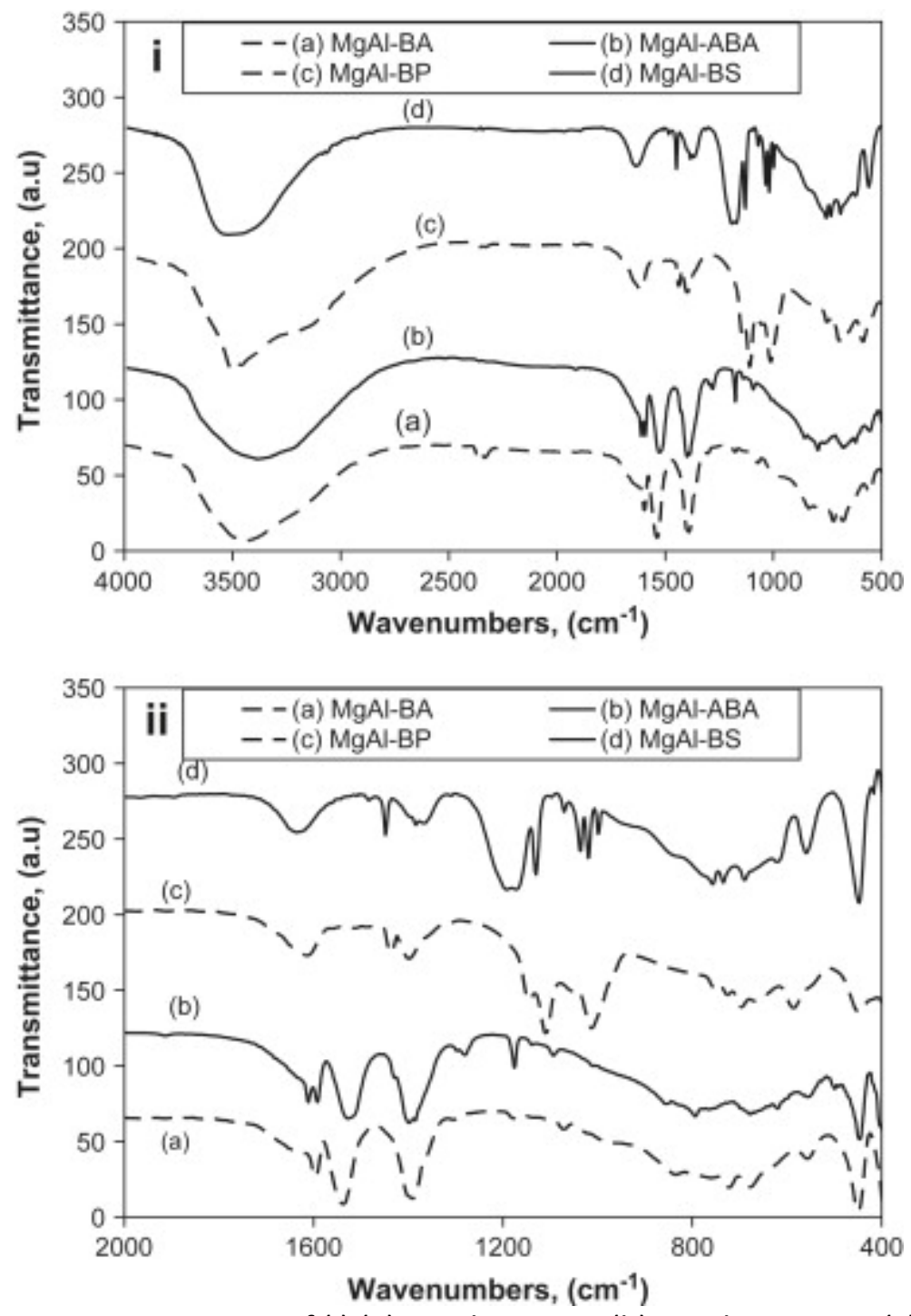

Fig. 2. FTIR spectra of (i) (a) MgAl-BA LDH, (b) MgAl-ABA LDH, (c) MgAl-BP LDH, and (d) MgAl-BS, (ii) expansion of FTIR spectra for the LDHs from 400 to $2000 \mathrm{~cm}^{-1}$. Spectra are offset for clarity.

Fig. $3(a)$ presents XRD traces and $2 \vartheta$ values of the MgAl-LDHs obtained from the coprecipitation method with the various benzyl anions. The XRD patterns show first and second order diffraction peaks which are equally spaced, which is characteristic of clays with a good layered structure. Using the Bragg equation, the dspacing of MgAl-BA, MgAl-ABA, MgAl-BS and MgAl-BP LDHs are $15.5 \AA, 15.0 \AA, 15.8 \AA$ and $14.2 \AA$, respectively. The differences in the $d$-spacing may be due to differences in the length and angle of tilt of the anions in the galleries of the LDHs. Fig. 3(b) shows the XRD traces of the MgAl-BP obtained using the different methods. After ion exchange of the MgAl-nitrate LDH, new diffraction peaks appear at lower values of $2 \vartheta$ indicating expansion of the galleries due to intercalation of the larger benzene phosphonate (BP) anions. In general, the sharpness and intensity of the diffraction peaks is considered to be proportional to crystallinity 16$]$. From Fig. 3(b), it is clear that the XRD pattern of the MgAl-BP obtained from the rehydration method gives sharper and stronger diffraction peaks, which indicates that the rehydration method gives the best crystallinity and this method may be the best way to preparing LDH with good crystallinity. There is no significant difference in the d-spacings of 
the MgAl-BP LDHs prepared using the various methods. FTIR and XRD analyses confirm the intercalation of the benzyl anions in the galleries of the MgAl-LDHs. The FTIR and XRD data are in good agreement with those reported in the literature [17], [18], [19].
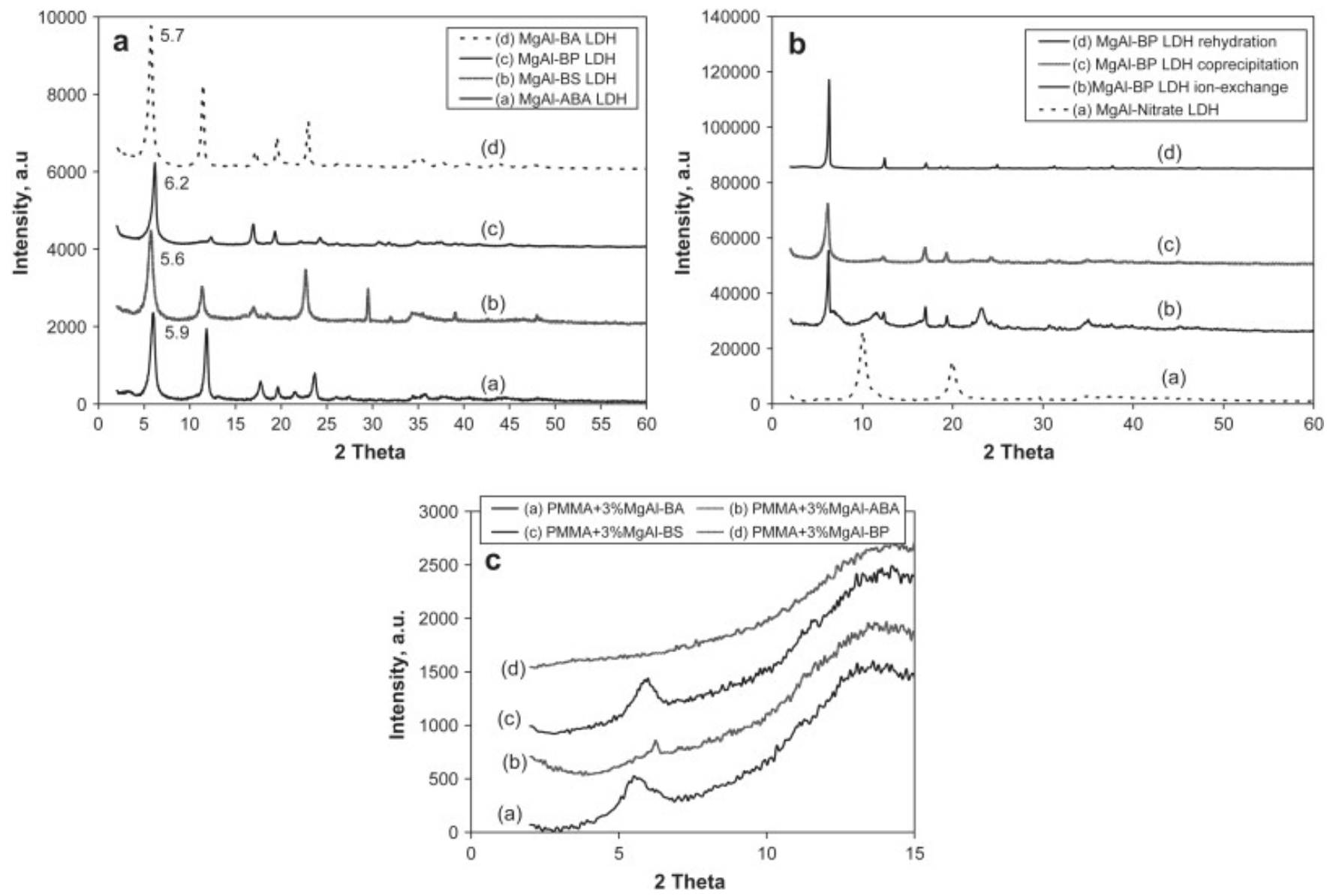

Fig. 3. (a): Powder XRD patterns of MgAl-LDHs obtained using the coprecipitation method. (b): XRD patterns of MgAl-LDHs prepared using different methods. (c): XRD patterns of PMMA composites.

\subsubsection{XRD analysis and transmission electron microscopy (TEM) of the PMMA composites}

The dispersion of the MgAl-LDHs was evaluated using XRD and TEM. Fig. 3(c) presents the XRD traces of the PMMA + MgAl-benzyl LDH composites while the TEM images are shown in Fig. 4. The basal spacing for MgAl$\mathrm{BA}$ and $\mathrm{MgAl}-\mathrm{BS}$ does not change, which may suggest microcomposite formation. No diffraction peaks are observed with the MgAl-ABA and MgAl-BP composites and this may suggest exfoliation or the LDH may be disordered. Further assessment of dispersion was obtained from TEM analyses. The higher magnification TEM images of MgAl-BA and MgAl-BP composites in Fig. 4(c) and (g) show good dispersion while those of the MgAl$\mathrm{BS}$ and MgAl-ABA show large agglomerates and poorly dispersed LDH particles. At higher magnification, the TEM images in Fig. 4(b), (d), (f) and (h) show that nano-dispersion has not been achieved and the MgAl-LDH composites may best be described as microcomposites, in good agreement with the observed XRD data of the composites. 

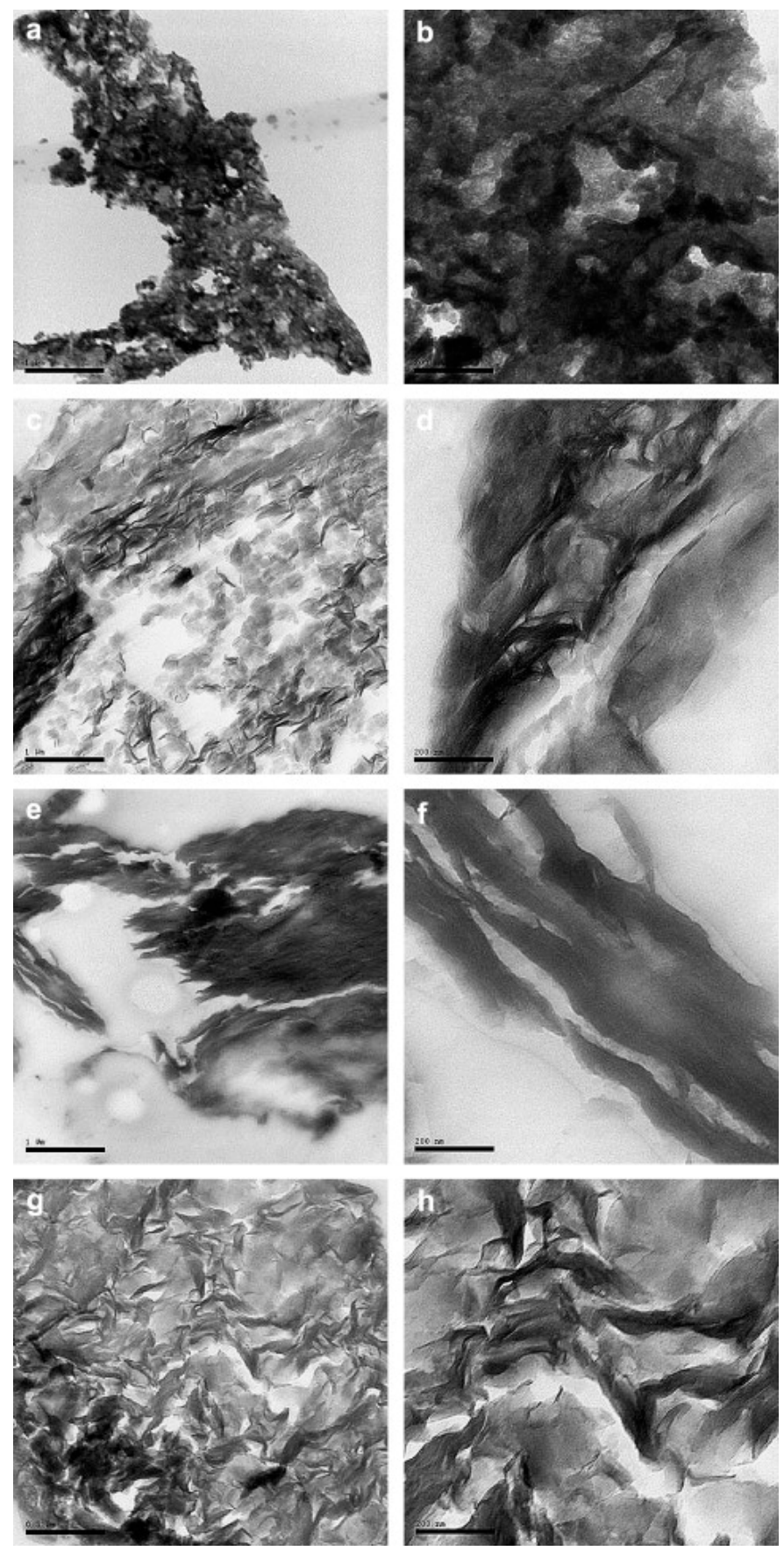

Fig. 4. TEM images at (a) low and (b) high magnification for PMMA + 3\% MgAl-ABA, (c) low and (d) high magnification for PMMA + 3\% MgAl-BA, (e) low and (f) high magnification for PMMA + 3\% MgAl-BS. The scale bars for low and high magnification represent $1000 \mathrm{~nm}$ and $200 \mathrm{~nm}$ distances respectively. TEM images at $(\mathrm{g})$ low and (h) high magnification for PMMA + 3\% MgAl-BP have scale bars of $500 \mathrm{~nm}$ and $200 \mathrm{~nm}$.

\subsection{Thermal stability of the MgAl-LDHs and their PMMA composites}

TGA curves of the LDHs are shown in Fig. 5(a)(i) and the data are summarized in Table 1. As is usual for $\mathrm{LDHs}$, the thermal degradation reveals three or four general regions of mass loss ${ }^{[20]}$. The weight loss up to $250{ }^{\circ} \mathrm{C}$ is ascribed to the loss of adsorbed and interlayer water. Unlike the first step, which is relatively rapid, the last two weight loss steps are very gentle and long. The steps overlap to some extent and involve dehydroxylation of the brucite-like layers followed by decomposition of the organic anions. The thermal degradation steps of the LDHs are clearly visible in the DTG curves presented in Fig. 5(a)(ii). From the summary 
of TGA data presented in Table 1, the MgAl-BA gives the lowest temperature at which $10 \%$ mass loss occurs $\left(T_{0.1}\right)$ and amount of residue at $600{ }^{\circ} \mathrm{C}$. There is no significant difference in the amount of residue for $\mathrm{MgAl}-\mathrm{BA}$ and MgAl-ABA, MgAl-BS and MgAl-BP obtained using the coprecipitation method. TGAs of MgAl-BP LDHs prepared using the various methods (i.e. coprecipitation, ion exchange and rehydration of calcined LDH) are similar, as shown in Fig. 5(b)(i) and (ii). The three methods also lead to the formation of the same amount of residue, 58\%, while the $T_{0.1}$ and the DTG plots show no significant differences. These observations suggest that LDHs prepared using different methods have the same thermal stability.
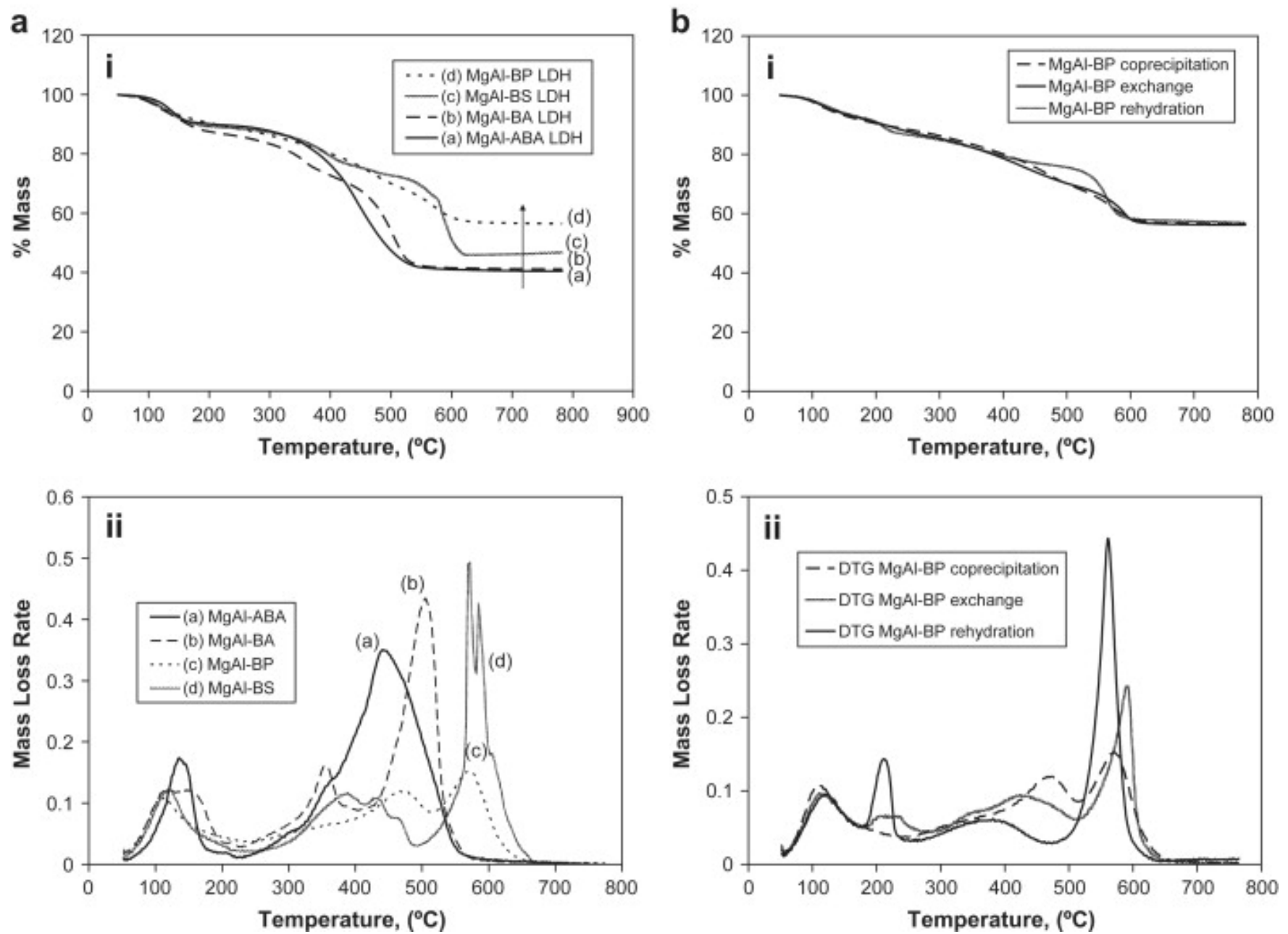

Fig. 5. (a): (i) TGA and (ii) DTG curves for MgAl-LDHs prepared using the coprecipitation method. (b): (i) TGA and (ii) DTG curves for MgAl-BP LDHs prepared using different methods.

Table 1. TGA data for the MgAl-LDHs prepared using different methods.

\begin{tabular}{|l|l|l|l|}
\hline Formulation & $\boldsymbol{T}_{\mathbf{0 . 1}}\left({ }^{\circ} \mathbf{C}\right)$ & $\boldsymbol{T}_{\mathbf{0 . 5}}\left({ }^{\circ} \mathbf{C}\right)$ & \% Char \\
\hline MgAl-BA LDH coprecipitation & 165 & 510 & 42 \\
\hline MgAl-ABA LDH coprecipitation & 206 & 489 & 41 \\
\hline MgAl-BS LDH coprecipitation & 226 & 682 & 55 \\
\hline MgAl-BP LDH coprecipitation & 210 & - & 59 \\
\hline MgAl-BP LDH exchange & 212 & - & 58 \\
\hline MgAl-BP LDH rehydration & 208 & - & 58 \\
\hline
\end{tabular}

$T_{0.1}$, temperature at which $10 \%$ mass loss occurs; $T_{0.5}$, temperature at which $50 \%$ mass loss occurs; $\%$ Char, residue at $600^{\circ} \mathrm{C}$. 
TGA curves for the PMMA composites at 3 and 10\% loading of the MgAl-LDHs are presented in Fig. 6(a) and the data are summarized in Table 2. Addition of the MgAl-BP/BA/ABA LDHs at low loading, $3 \mathrm{wt} \%$, improves the thermal stability significantly, whether $T_{0.1}$ or $T_{0.5}$ are used as the point of comparison. Increasing the loading of the LDH to $10 \%$ results in a further increase in thermal stability and the amount of char. With the MgAl-BS, a reverse effect of loading on thermal stability is observed. A loading of $3 \% \mathrm{MgAl}-\mathrm{BS}$ results in more improvement in thermal stability than is obtained at $10 \%$ loading but the amount of char/residue is increased. The thermal stability of the MgAl-BS LDH was better than that of MgAl-BA and MgAl-ABA, as shown by the TGA and DTG data in Fig. 5 and Table 1. This suggests that an LDH with higher thermal stability does not always indicate that enhanced thermal stability can be expected when it is combined with a polymer. TGA curves showing a comparison at $10 \%$ loading of MgAl-LDHs prepared using coprecipitation in Fig. 6 (b)(i) show that the composite containing $10 \% \mathrm{MgAl}-\mathrm{BS}$ had the least thermal stability while there is no significant difference between the composites prepared using $10 \%$ of MgAl-BP LDHs obtained from the various methods, as shown in Fig. 6(b)(ii).
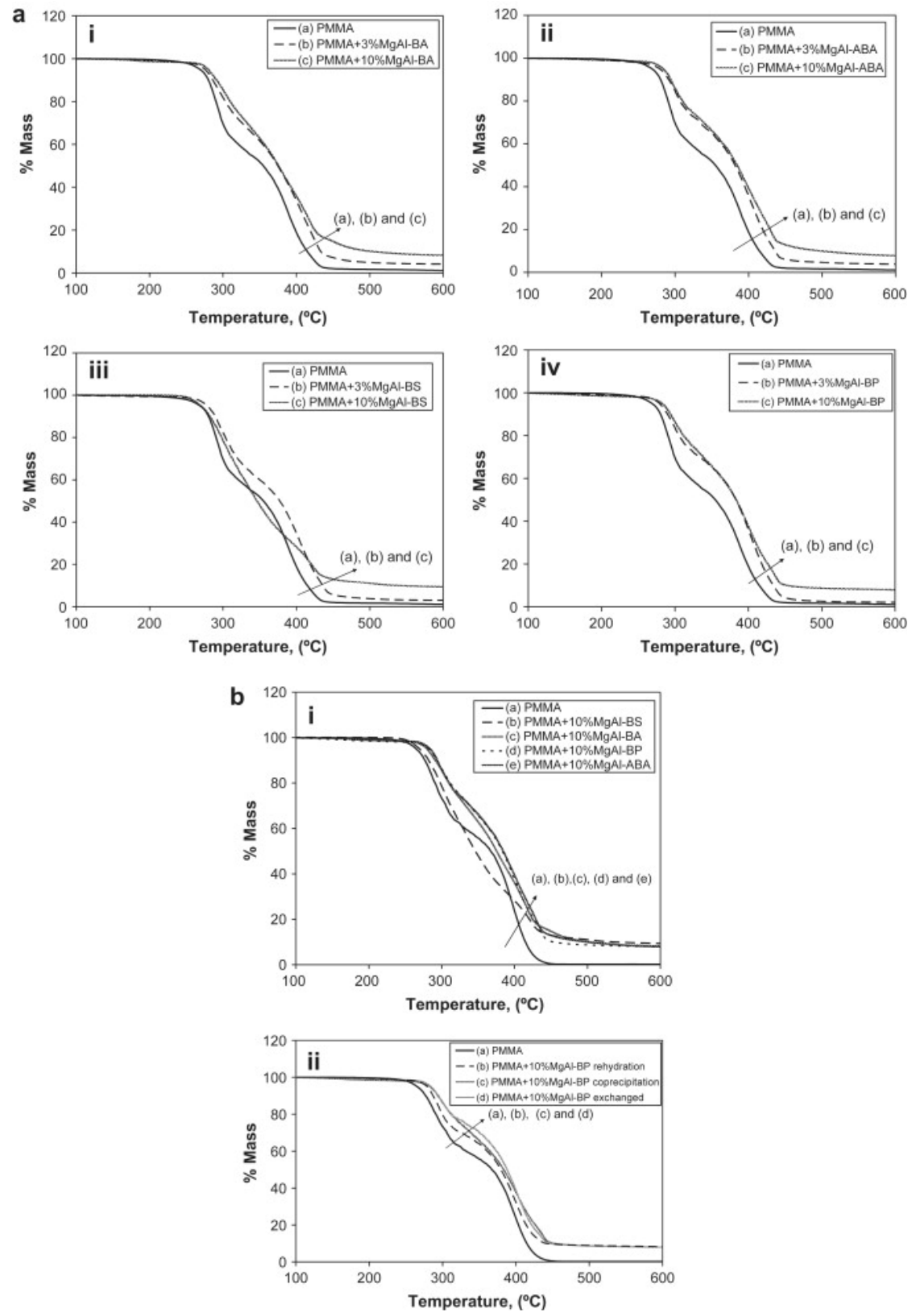

Fig. 6. (a): TGA curves (i) PMMA + MgAl-BA LDH, (ii) PMMA + MgAl-ABA LDH, (iii) PMMA + MgAl-BS LDH and (iv) PMMA + MgAl-BP LDH prepared using the coprecipitation method. (b): A comparison of TGA curves for (i) PMMA + 10\% MgAl-LDH from coprecipitation and (ii) PMMA + MgAl-BP prepared using different methods. 
Table 2. TGA data for poly(methyl methacrylate) and its composites.

\begin{tabular}{|c|c|c|c|c|c|}
\hline Formulation & $T_{0.1}\left({ }^{\circ} \mathrm{C}\right)$ & $T_{0.5}\left({ }^{\circ} \mathrm{C}\right)$ & $\%$ Char & $\Delta T_{0.5}$ & $\Delta T_{0.1}$ \\
\hline PMMA & 277 & 355 & 0 & - & - \\
\hline PMMA + 3\% MgAl-BA & 286 & 373 & 4 & 18 & 9 \\
\hline PMMA + 10\% MgAl-BA & 289 & 376 & 8 & 21 & 12 \\
\hline PMMA + 3\% MgAl-ABA & 291 & 382 & 4 & 27 & 14 \\
\hline PMMA + 10\% MgAl-ABA & 295 & 385 & 8 & 30 & 18 \\
\hline PMMA + 3\% MgAl-BS & 289 & 377 & 3 & 22 & 12 \\
\hline PMMA + 10\% MgAl-BS & 282 & 348 & 9 & -7 & 5 \\
\hline PMMA + 3\% MgAl-BP coprecipitation & 290 & 383 & 2 & 28 & 13 \\
\hline PMMA + 10\% MgAl-BP coprecipitation & 293 & 384 & 8 & 29 & 16 \\
\hline PMMA + 10\% MgAl-BP exchange & 295 & 389 & 8 & 34 & 18 \\
\hline PMMA + 10\% MgAl-BP rehydration & 286 & 379 & 8 & 24 & 9 \\
\hline
\end{tabular}

$T_{0.1}$, temperature at which $10 \%$ mass loss occurs; $T_{0.5}$, temperature at which $50 \%$ mass loss occurs; $\%$ Char, residue at $600{ }^{\circ} \mathrm{C} ; \Delta T_{0.5}, T_{0.5}$ (composites) $-T_{0.5}$ (neat PMMA); $\Delta T_{0.1}, T_{0.1}$ (composites) $-T_{0.1}$ (neat PMMA).

\subsection{Flammability behavior of the PMMA composites}

The cone calorimeter was used to assess the flammability behavior and this provided detailed information on the effectiveness of the MgAl-LDHs as fire retardants during combustion. The heat release rate (HRR), total heat released (THR), volume of smoke released (VOS), average mass loss rate (AMLR) and time-toignition ( $\left.t_{\mathrm{ig}}\right)$ are summarized in $\underline{\text { Table } 3}$ and the HRR curves are shown in Fig. $7(\mathrm{a})$ and (b).

Table 3. Cone calorimetry data for PMMA composites at $50 \mathrm{~kW} / \mathrm{m}^{2}$.

\begin{tabular}{|c|c|c|c|c|c|c|c|}
\hline Formulation & $\begin{array}{l}\text { PHRR } \\
\left(\mathrm{kW} / \mathrm{m}^{2}\right)\end{array}$ & $\begin{array}{l}\text { Rdtn } \\
\text { (\%) }\end{array}$ & $\begin{array}{l}\text { THR } \\
\left(\mathrm{MJ} / \mathrm{m}^{2}\right)\end{array}$ & $\operatorname{vos}(\mathrm{L})$ & $\begin{array}{l}\text { AMLR } \\
\left(\mathrm{g} / \mathrm{s} \mathrm{m}^{2}\right)\end{array}$ & $t_{\mathrm{ig}}(\mathrm{s})$ & $\begin{array}{l}\text { Char } \\
\text { (\%) }\end{array}$ \\
\hline PMMA & $1028 \pm 48$ & NA & $82 \pm 2$ & $412 \pm 51$ & $34 \pm 3$ & $14 \pm 1$ & $0 \pm 0$ \\
\hline PMMA + 3\% MgAl-BA LDH & $738 \pm 27$ & 28 & $76 \pm 1$ & $393 \pm 19$ & $26 \pm 1$ & $10 \pm 2$ & $4 \pm 0$ \\
\hline PMMA + 10\% MgAl-BA LDH & $554 \pm 35$ & 46 & $71 \pm 1$ & $598 \pm 32$ & $16 \pm 3$ & $14 \pm 1$ & $10 \pm 1$ \\
\hline PMMA + 3\% MgAl-ABA LDH & $828 \pm 34$ & 19 & $79 \pm 1$ & $292 \pm 77$ & $28 \pm 3$ & $9 \pm 2$ & $4 \pm 1$ \\
\hline $\begin{array}{l}\text { PMMA + 10\% MgAl-ABA } \\
\text { LDH }\end{array}$ & $665 \pm 6$ & 35 & $74 \pm 1$ & $403 \pm 76$ & $21 \pm 2$ & $12 \pm 2$ & $10 \pm 2$ \\
\hline PMMA + 3\% MgAl-BS LDH & $795 \pm 21$ & 23 & $77 \pm 1$ & $267 \pm 83$ & $30 \pm 1$ & $11 \pm 2$ & $3 \pm 0$ \\
\hline PMMA + 10\% MgAl-BS LDH & $736 \pm 42$ & 26 & $74 \pm 1$ & $226 \pm 64$ & $27 \pm 2$ & $12 \pm 3$ & $11 \pm 1$ \\
\hline PMMA + 3\% MgAl-BP LDH & $819 \pm 58$ & 20 & $78 \pm 1$ & $159 \pm 27$ & $29 \pm 1$ & $10 \pm 1$ & $4 \pm 1$ \\
\hline $\begin{array}{l}\text { PMMA + 10\% MgAl-BP } \\
\text { coprecipitation }\end{array}$ & $821 \pm 82$ & 20 & $75 \pm 2$ & $266 \pm 23$ & $28 \pm 1$ & $11 \pm 3$ & $14 \pm 1$ \\
\hline $\begin{array}{l}\mathrm{PMMA}+10 \% \mathrm{MgAl}-\mathrm{BP} \\
\text { rehydrated }\end{array}$ & $716 \pm 54$ & 30 & $75 \pm 2$ & $173 \pm 64$ & $24 \pm 1$ & $11 \pm 1$ & $12 \pm 1$ \\
\hline $\begin{array}{l}\text { PMMA + 10\% MgAl-BP } \\
\text { exchanged }\end{array}$ & $718 \pm 69$ & 30 & $77 \pm 3$ & $241 \pm 179$ & $25 \pm 2$ & $11 \pm 2$ & $13 \pm 1$ \\
\hline
\end{tabular}

PHRR, peak heat release rate; (\% rdtn), reduction in PHRR; THR, total heat release; VOS, volume of smoke released; AMLR, average mass loss rate; $t_{\text {ign, }}$ time-to-ignition; Char, $\%$ residue after cone calorimetry. 

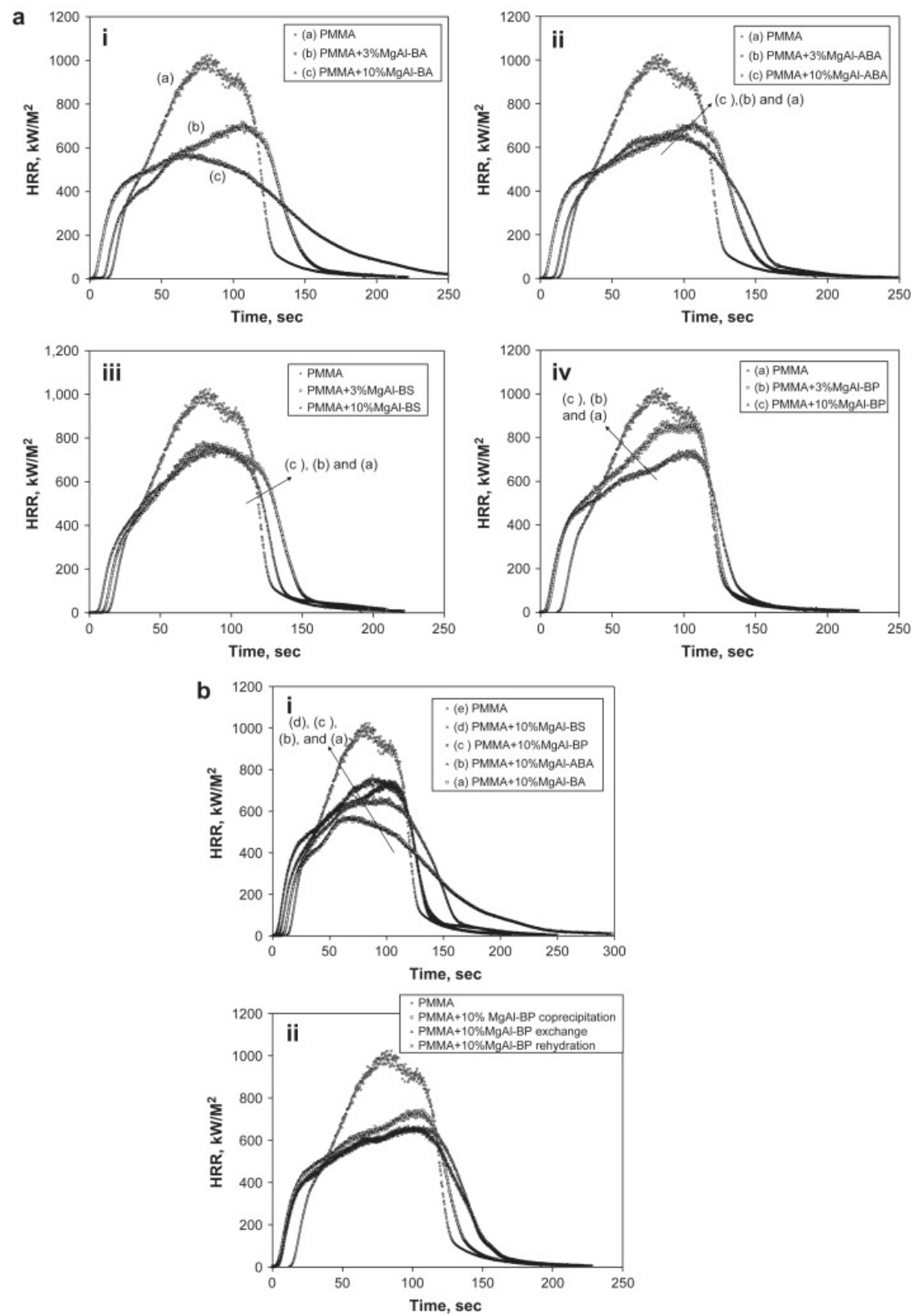

Fig. 7. (a): Heat release rate curves for (i) PMMA + MgAl-BA LDH, (ii) PMMA + MgAl-ABA LDH, (iii) PMMA + MgAlBS LDH, and (iv) PMMA + MgAl-BP. The LDHs were prepared using the coprecipitation method. (b): Heat release rate curves for (i) PMMA + 10\% MgAl-LDHs, prepared using the coprecipitation method and (ii) PMMA + 10\% MgAl-BP LDHs prepared using various methods.

From the data, one can see that the addition of the MgAl-LDHs significantly reduces the PHRR and AMLR and the reduction in both the PHRR and AMLR is higher at $10 \%$ loading than at $3 \%$. It has been shown with montmorillonite that the reduction in the PHRR corresponds to a reduction in the mass loss rate, and this is also observed in these LDH systems. For the LDHs prepared using the coprecipitation method, the best reductions, $46 \%$ and $35 \%$, are obtained with 10\% MgAl-BA and 10\% MgAl-ABA while the systems with MgAl-BP and MgAl-BS give reductions that are much lower, $20 \%$ and $26 \%$, respectively. Based on the HRR values, there is no significant differences in the fire retardant effectiveness of the LDHs prepared using different methods (i.e. coprecipitation, ion exchange and rehydration methods). The char yield increases as the loading of the LDH increase from 3 to $10 \%$ and this is expected as the char is mostly LDH residue. The fire retardant action of LDHs may be attributed to: (i) dilution and cooling of flammable volatiles by non-flammable gases such as water and $\mathrm{CO}_{2}$ evolved from the thermal degradation of LDHs, (ii) the formation of refractory oxides over the polymer surface that acts as a 
barrier for heat and mass transfer and (iii) the endothermic decomposition of the LDHs that results in the absorption of heat from the burning polymer, hence reducing the thermal degradation of the polymer and the flaming process $\stackrel{[21]}{2}$.

Photographs of the char after cone calorimetry are presented in Fig. 8. Char formation is an important aspect of fire retardancy [22]. In general, an increase in char formation is associated with a reduction of HRR and is an indication of good fire retardant effectiveness. The char obtained with composites containing $10 \%$ loading of MgAl-ABA, MgAl-BS and MgAl-BP consists of several separated small hard particles. Surprisingly, the char particles obtained with MgAl-BA were expanded, large, soft, and airy and showed evidence of blowing. Expanded char provides a very effective barrier for slowing heat and mass and mass transfer [23], [24], which may explain why the best reduction in the PHRR was observed with MgAl-BA LDH.
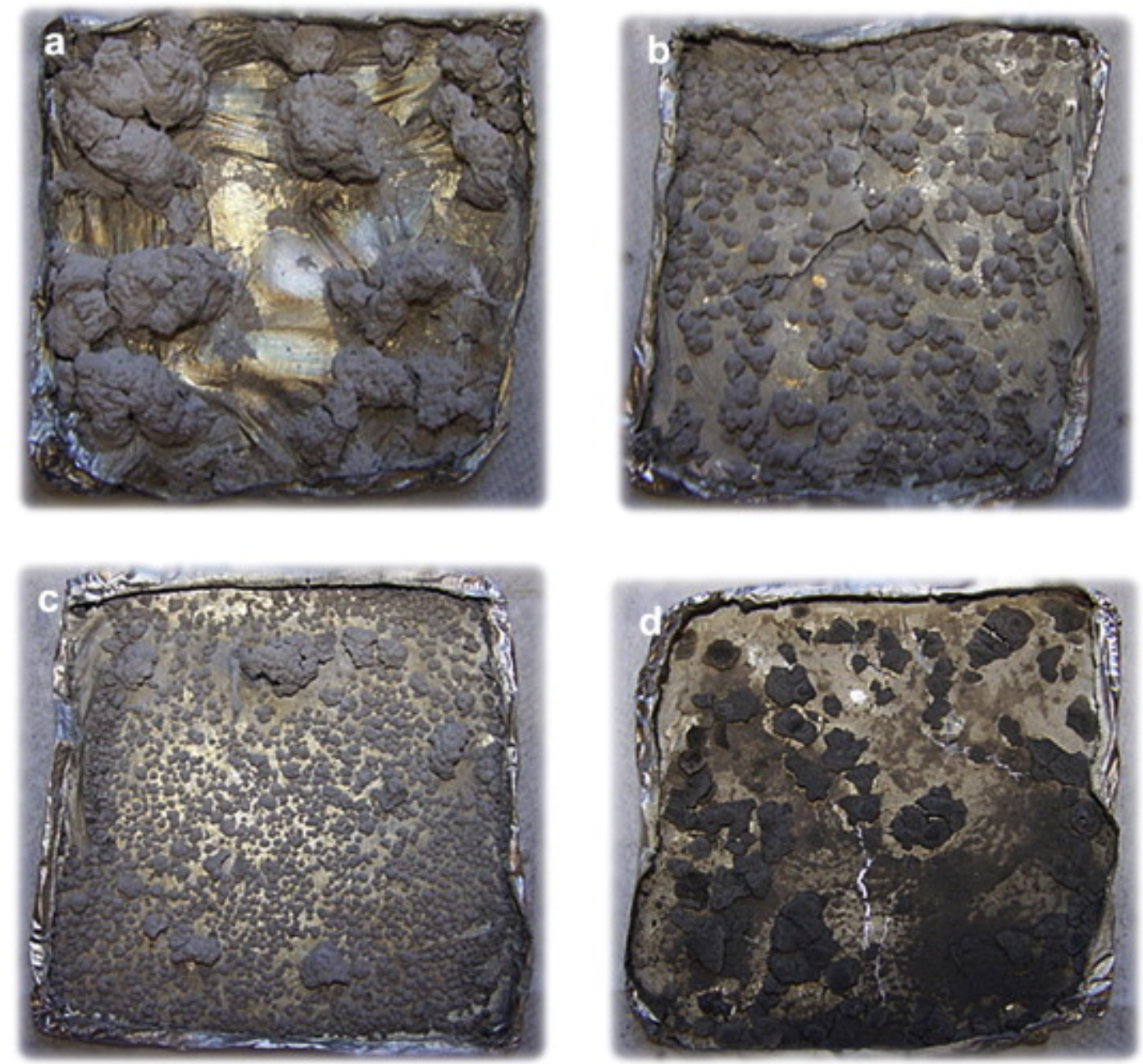

Fig. 8. Photographs of residual char from (a) PMMA + MgAl-BA LDH, (b) PMMA + MgAl-ABA LDH, (c)

PMMA + MgAl-BS LDH, and (d) PMMA + MgAl-BP formulations following cone calorimetry test under a heat flux of $50 \mathrm{~kW} / \mathrm{m}^{2}$.

XRD patterns for the char obtained after cone calorimetry are non-descript and show no evidence for formation of crystalline metal oxide phases. Calcination of the char at $1000^{\circ} \mathrm{C}$ resulted in the formation of

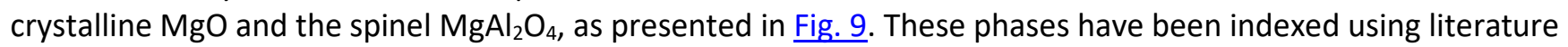

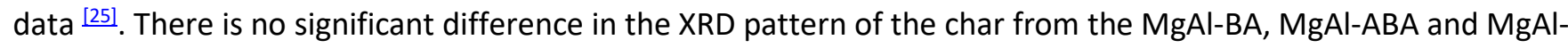
BS systems, but the XRD traces of the MgAl-BP showed low intensities of the $\mathrm{MgO}$ and $\mathrm{MgAl}_{2} \mathrm{O}_{4}$ indicating reduced crystallinity of these phases. These same phases have been previously observed in cone residue with MgAl-LDHs $\stackrel{[26]}{ }$. 


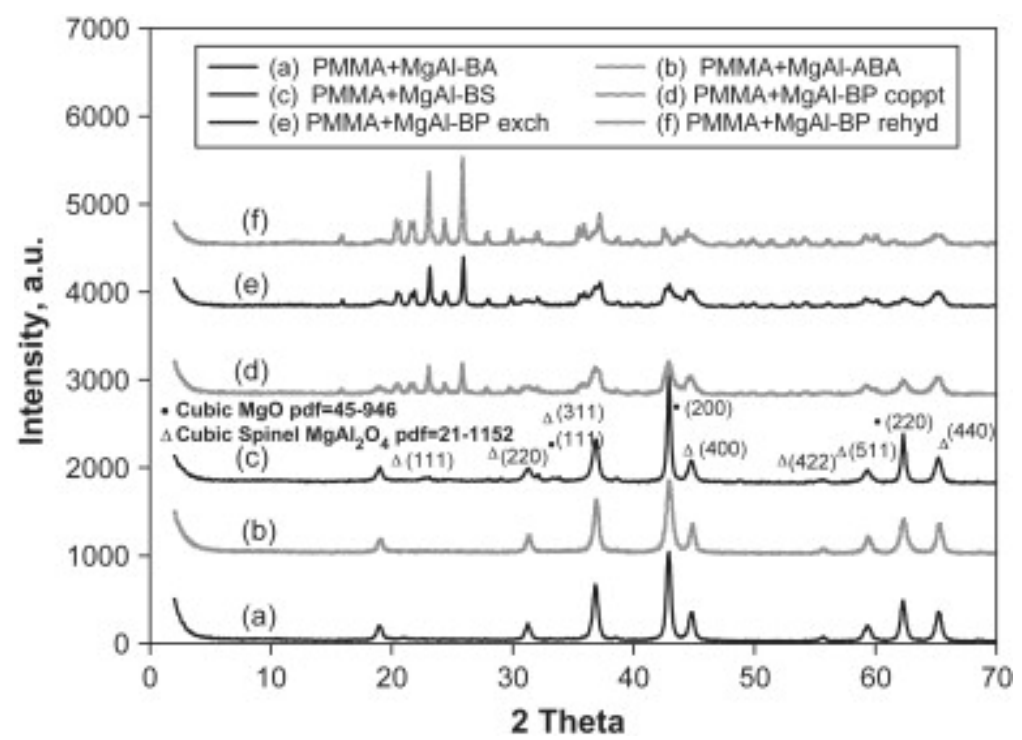

Fig. 9. XRD of residual char for; (a) PMMA + MgAl-BA LDH, (b) PMMA + MgAl-ABA LDH, (c) PMMA + MgAl-BS $\mathrm{LDH}$, and (d) PMMA + MgAl-BP, formulations following cone calorimetry test under a heat flux of $50 \mathrm{~kW} / \mathrm{m}^{2}$.

\section{Conclusions}

MgAl-LDHs intercalated with various benzyl anions were prepared using various methods.

Characterization of the LDHs by TGA and FTIR show no significant differences in the method of preparation while XRD analyses reveals high crystallinity for the LDH prepared using the rehydration of calcined LDH. The MgAlLDHs were melt-blended with PMMA to prepare composites which were characterized using cone calorimetry and TGA. Addition of the LDHs to PMMA improved the thermal stability of the composites. The least thermal stabilization effect was observed in composites containing MgAl-BS. No significant differences in the thermal stability of composites with LDH prepared using different methods were observed. TEM shows that the MgAlLDH systems were microcomposites. Fire properties evaluated using the cone calorimeter indicates that the PHRR is significantly reduced when the MgAl-LDHs are added to PMMA. The best reduction of $46 \%$ was observed in the PMMA + 10\% MgAl-BA system. Composites with LDHs prepared using the different methods showed no significant differences in the HRR. The improved fire performance of the composites was attributed to the endothermic thermal decomposition process of the $\mathrm{LDH}$ that release $\mathrm{CO}_{2}$ and $\mathrm{H}_{2} \mathrm{O}$ which cools and dilutes the flaming volatiles and also results in the formation of mixed metal oxides that act as barrier for heat and mass transfer. XRD analyses obtained after cone calorimetry show that the oxides formed during combustion were amorphous while calcination of the char resulted in the formation of crystalline $\mathrm{MgO}$ and $\mathrm{MgAl}_{2} \mathrm{O}_{4}$ spinel phases.

\section{Acknowledgements}

The authors gratefully thank Dr. Jeanne Hossenlopp and Dr. Walid Awad for helpful discussions. Partial support of this work was provided by the US Department of Commerce, National Institute of Standard and Technology under grant number 60NANB6D6018.

Recommended articlesCiting articles (36)

\section{References}

[1] C. Nyambo, D. Wang, C.A. Wilkie Will layered double hydroxides give nanocomposites with polar or nonpolar polymers? Polym Adv Technol (2008) doi:10.1002/pat.1272

[2] C. Nyambo, P. Songtipya, E. Manias, M. Jimenez-Gasco, C.A. Wilkie Effect of MgAl-layered double hydroxide exchanged with linear alkyl carboxylates on fire retardancy of PMMA and PS J Mater Chem, 18 (2008), pp. 4827-4838 
[3] D.G. Evans, X. Duan Preparation of layered double hydroxides and their applications as additives in polymers, as precursors to magnetic materials and in biology and medicine Chem Commun, 5 (2006), pp. $485-496$

[4] C. Nyambo, E. Kandare, D. Wang, C. Wilkie Flame-retarded polystyrene: investigating chemical interactions between ammonium polyphosphate and MgAl layered double hydroxide Polym Degrad Stab, 93 (2008), pp. 1656-1663

[5] F. Cavani, F. Trifiro, A. Vaccari Hydrotalcite type anionic clays preparation, properties and applications Catal Today, 11 (1991), pp. 173-301

[6] J. He, M. Wei, B. Li, Y. Kang, D.G. Evan, X. Duan Preparation of layered double hydroxides Struct Bonding, 119 (2006), pp. 89-119

[7] S.P. Newman, W. Jones Synthesis, characterization and applications of layered double hydroxides containing organic guests New J Chem, 2 (1998), pp. 105-115

[8] S. Carlino The intercalation of carboxylic acids into layered double hydroxides: A critical evaluation and review of different methods Solid State lonics, 98 (1997), pp. 73-84

[9] A. De Roy, C. Forano, J.P. Besse Layered double hydroxides: synthesis and post synthesis modification Vicente Rives (Ed.), Layered double hydroxides; present and future, Nova Science Publisher (2001), pp. $115-137$

[10] S. Carlino, M. Hudson Thermal intercalation of layered double hydroxides: capric acid into an MgAl-LDH J Mater Chem, 5 (1995), pp. 1433-1442

[11] S. Carlino, M.J. Hudson Reaction of molten sebacic acid with a layered (Mg/Al) double hydroxide J Mater Chem, 4 (1994), pp. 99-104

[12] E.D. Dimotakis, T.J. Pinnavaia New route to new layered double hydroxides intercalated by organic anions: precursors to polyoxometalate pillered derivatives Inorg Chem, 29 (1990), pp. 2393-2394

[13] G.A. Wang, C.C. Wang, Y.C. Chen The disorderly exfoliated LDHs/PMMA nanocomposite synthesized by in situ bulk polymerization Polymer, 46 (2005), p. 5065

[14] M. Meyn, K. Benecke, G. Lagally Anion-exchange reactions of layered double hydroxides Inorg Chem, 29 (1990), pp. 5201-5207

[15] D. Wang, J. Zhu, Q. Yao, C.A. Wilkie A comparison of various methods for the preparation of polystyrene and poly(methyl methacrylate) clay nanocomposites Chem Mater, 14 (2002), pp. 3837-3843

[16] U. Costantino, M. Nocchetti, F. Marmottini, R. Vivani Amino acid derivatives of layered zirconium phosphates $-\alpha$-zirconium I-(+)-serinephosphate and zirconium I-(+)-serinephosphate phosphates Eur J Inorg Chem, 10 (1998), pp. 1447-1452

[17] V. Prevot, C. Forano, J.P. Besse Hybrid derivatives of layered double hydroxides Appl Clay Sci, 18 (2001), pp. 3-15

[18] H.B. Hsueh, C.-Y. Chen Preparation of LDHs/polyimide nanocomposites Polymer, 44 (2003), pp. 1151-1161

[19] G.R. Williams, G. O'Hare New phosphonate intercalates of $\left[\mathrm{Ca}_{2} \mathrm{Al}(\mathrm{OH})_{6}\right] \mathrm{NO}_{3} \cdot y \mathrm{H}_{2} \mathrm{O}$ : a kinetic study Solid State lonics, 8 (2006), pp. 971-980

[20] L. Pesic, S. Salipurovic, V. Markovic, D. Vucelic, W. Kagunya, W. Jones Thermal characteristics of a synthetic hydrotalcite-like material J Mater Chem, 2 (1992), pp. 1069-1073

[21] M. Zammarano, M. Franceshi, Bellayer, J.W. Gilman, S. Meriani Preparation and flame resistance properties of revolutionary self-extinguishing epoxy nanocomposites based on layered double hydroxides Polymer, 46 (2005), pp. 9314-9328

[22] S. Levchik, C.A. Wilkie Char formation A.F. Grand, C.A. Wilkie (Eds.), Fire retardancy of polymeric materials, Marcel Derker Inc. (2000), pp. 171-215

[23] M. Jimenez, S. Bourbigot, S. Duquesne Intumescent fire protective coating: toward a better understanding of their mechanism of action Thermochim Acta, 449 (2006), pp. 16-26

[24] S. Bourbigot, M. Le Bras, S. Duquesne, M. Rochery Recent advances in intumescent polymers Macromol Mater Eng, 289 (2004), pp. 499-511

[25] Powder diffraction file alphabetical indexes, inorganic phases, pdf = 21-1152 JCPDS International Center for Diffraction Data, Swartmore, PA (1999) 
[26] Manzi-Nshuti C, Songtipya P, Manias E, Jimenez-Gasco MM, Hossenlopp JM, Wilkie CA. Polymer nanocomposites using zinc aluminum and magnesium aluminum oleate layered double hydroxides: effects of LDH divalent metals on dispersion, thermal, mechanical and fire properties in various polymers, submitted for publication. 